\title{
Dendritic Amputation Redistributes Sprouting Evoked by Axotomy in Lamprey Central Neurons
}

\author{
Garth F. Hall and Melvin J. Cohen \\ Department of Biology, Yale University, New Haven, Connecticut 06511
}

In the previous paper (Hall and Cohen, 1988), we showed that axotomy of anterior bulbar cells (ABCs) in the hindbrain of the larval lamprey results in the sprouting of axonlike neurites from either the end of the proximal axon stump, the dendritic tips, or both, depending on the site of axotomy. Here we show that, unlike axotomy, dendritic amputation (dendrotomy) does not by itself induce sprouting from ABCs. However, dendrotomy does induce sprouting from dendrites in the immediate vicinity of the dendritic lesion in cells that have been previously axotomized. We found that dendrotomy acts primarily to rearrange the distribution of sprouts induced by axotomy rather than serving as an additional stimulus to neurite outgrowth.

We propose that (1) dendritic sprouting in ABCs occurs because the dendritic tips become attractive sites for sprout initiation when they are either directly injured (as with dendrotomy) or are situated relatively close to the site of injury (as with axotomy close to the soma), and (2) the axon stump, dendritic stumps, and uninjured dendritic tips of the cell compete to initiate a limited total amount of sprouting induced by axotomy. The probability that a given locus will support sprouting is determined both by its proximity to the nearest lesion site and by whether there are other attractive potential sprouting sites in the cell.

Neuritic sprouting from mature neurons in vivo can be induced by several types of injury in a variety of neuronal systems. Sprouting can be caused either by direct injury to the neuron (usually axotomy) or by indirect effects of injury such as deprivation or expansion of available postsynaptic targets (Aguilar et al., 1973) or deafferentation (Hoy et al., 1978; Pitman and Rand, 1982). In gencral, howcver, profusc sprouting is only induced by axotomy, and it usually takes the form of axonal regeneration from the cut end of the axon stump. Recently, there have been a few reports of profuse neuritic sprouting from sites other than the axon stump following axotomy of identified invertebrate interneurons (Murphy and Kater, 1980; Roederer and Cohen, 1983; Schacher and Proshansky, 1983). Some dendritic sprouting in response to axotomy has also been reported in a vertebrate, the sea lamprey, both in Muller interneurons in the hindbrain (Hall and Cohen, 1983, 1988) and in giant inter-

\footnotetext{
Received Dec. 31, 1986; revised Feb. 2, 1988; accepted Feb. 9, 1988.

We thank Ann Poulos for her technical assistance and Haig Keshishian and Sylvie Clausse for their helpful criticism of the manuscript. This work was supported by NIH Spinal Trauma Grant 2P50 NS 10174-14 and NIH Physiology Training Grant GM 15727.

Correspondence should be addressed to Garth F. Hall, Department of Zoology, University of California, Berkeley, CA 94720.

Copyright (C) 1988 Society for Neuroscience 0270-6474/88/103598-09\$02.00/0
}

neurons in the spinal cord (Yin et al., 1984; Mackler et al., 1986)

In previous studies, we have described profuse neuritic sprouting from the dendrites and/or the proximal axon stump in a group of giant identified central neurons (ABCs) in the larval lamprey following axotomy at various distances from the soma (Hall and Cohen, 1983, 1988). Neuritic sprouts originating from the dendritic tips are similar to axonal sprouts in their gross morphology, ultrastructure (Hall et al., 1987), and trajectories. Furthermore, dendritic and axonal sprouting appears to be triggered by a common mechanism set in motion by axotomy. Thus, at this level of analysis, the only obvious difference between dendritic and axonal sprouts is their site of origin within the neuron. These results raise 2 questions. (1) Is axotomy the only type of direct injury that will elicit sprouting from ABCs? (2) What are the factors that detcrminc where sprouts cmcrge from these neurons following axotomy? These questions are addressed in this paper, where we present the results of experiments in which $\mathrm{ABCs}$ were subjected to dendritic amputation (dendrotomy) alone and in combination with axotomy.

\section{Materials and Methods}

Operations. Larval lampreys $10 \mathrm{~cm}$ or more in length were anesthetized and the hindbrain opened as described previously (Hall and Cohen, 1988). All lesioning was performed with a No. 11 scalpel blade. "Distant" axotomy lesions were produced by a complete transection of the spinal cord at the level of the fifth gill $1-1.5 \mathrm{~cm}$ away from the $\mathrm{ABC}$ somata (Hall and Cohen, 1988). An axotomy site approximately 750 $\mu \mathrm{m}$ from the ABC somata was also used in some experiments. Amputation of the distal portion of the lateral dendrite (dendrotomy) was accomplished with a sagittal lesion located immediately medial of the sulcus limitans of His and extending through the entire depth of the brain (Fig. 1). This lesion extended for several hundred microns beyond the rostral and caudal limit of the $\mathrm{ABC}$ dendritic field, producing extensive damage to local tissues and partial deafferentation of the ABCs as well as dendrotomy. The precise location of the lesion was critical, as lesions within $75 \mu \mathrm{m}$ of the $\mathrm{ABC}$ somata usually killed the cells, while lesions further than $150 \mu \mathrm{m}$ from the somata did not remove enough of the dendrite for dendrotomy to be confirmed by subsequent intracellular staining with Lucifer yellow.

The filling of $\mathrm{ABCs}$ with Lucifer yellow, and the histological procedures used to examine filled cells in wholemount and in serial sections are identical to those described in the accompanying paper (Hall and Cohen, 1988).

\section{Results}

Dendritic stability following dendrotomy of intact $A B C s$

Amputation of the distal portions of the lateral dendritic tree did not induce major changes in the gross morphology of otherwise intact ABCs (Fig. 1). Only one cell of 61 cells examined between 19 and $220 \mathrm{~d}$ after dendrotomy exhibited any evidence of sprouting from the dendrites. No cells showed any signs of 

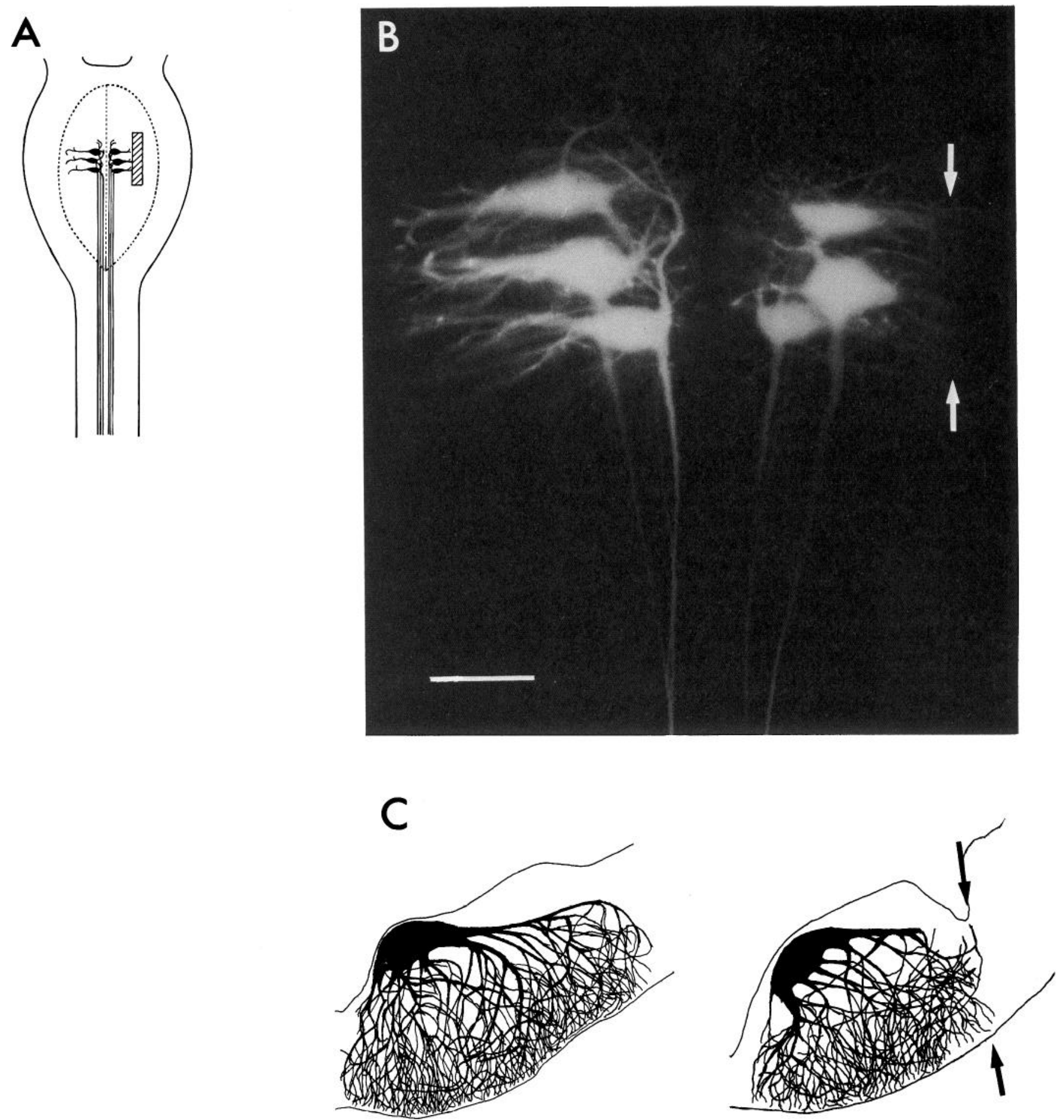

Figure 1. Morphological stability of $\mathrm{ABC}$ dendrites following dendrotomy. $A$, Schematic diagram of the lamprey hindbrain showing the location of anterior bulbar cells (ABCs) and the site of lateral dendrotomy (hatched box). B. Three pairs of ABCs $27 \mathrm{~d}$ after lateral dendrotomy of the cells on the right side. Cells on the left are intact and serve as controls. A rostrocaudally oriented lesion slightly medial to the lateral limit of the ABCs (arrow) amputated the distal portion of the lateral dendrites. Dendrotomy never produced significant sprouting from or retraction of the dendrites. $C$, Reconstructions from serial sections of a normal ABC (left) and a cell $112 \mathrm{~d}$ after dendrotomy (right). Arrows indicate the lesion site. Note that there is no dendritic retraction; fine dendrites still extend to the ventral margin of the brain, in contrast to the retraction of these dendrites seen at the same time postaxotomy (Hall and Cohen, 1988). Scale bar, $100 \mu \mathrm{m}$.

dendritic retraction. A few cells showed some shrinkage of their somata following dendrotomy, although this was never accompanied by dendritic retraction (as described in Hall and Cohen, 1988).
This stability of the dendrites in response to direct injury is in sharp contrast to the extensive sprouting and subsequent retraction of the dendrites evoked by "close" axotomy at a point within $500 \mu \mathrm{m}$ of the soma (Hall and Cohen, 1983, 1988). This 
A

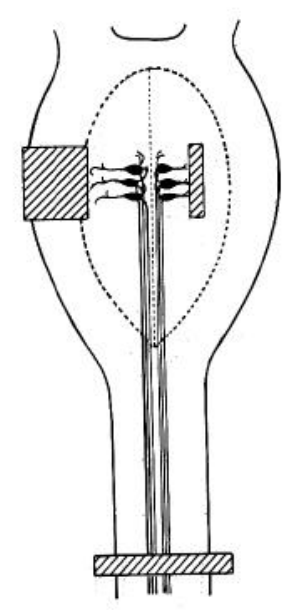

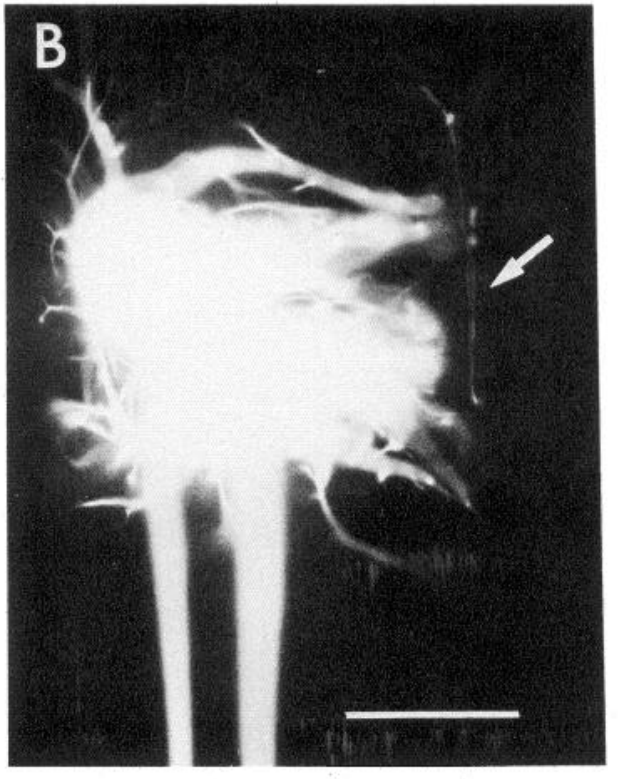
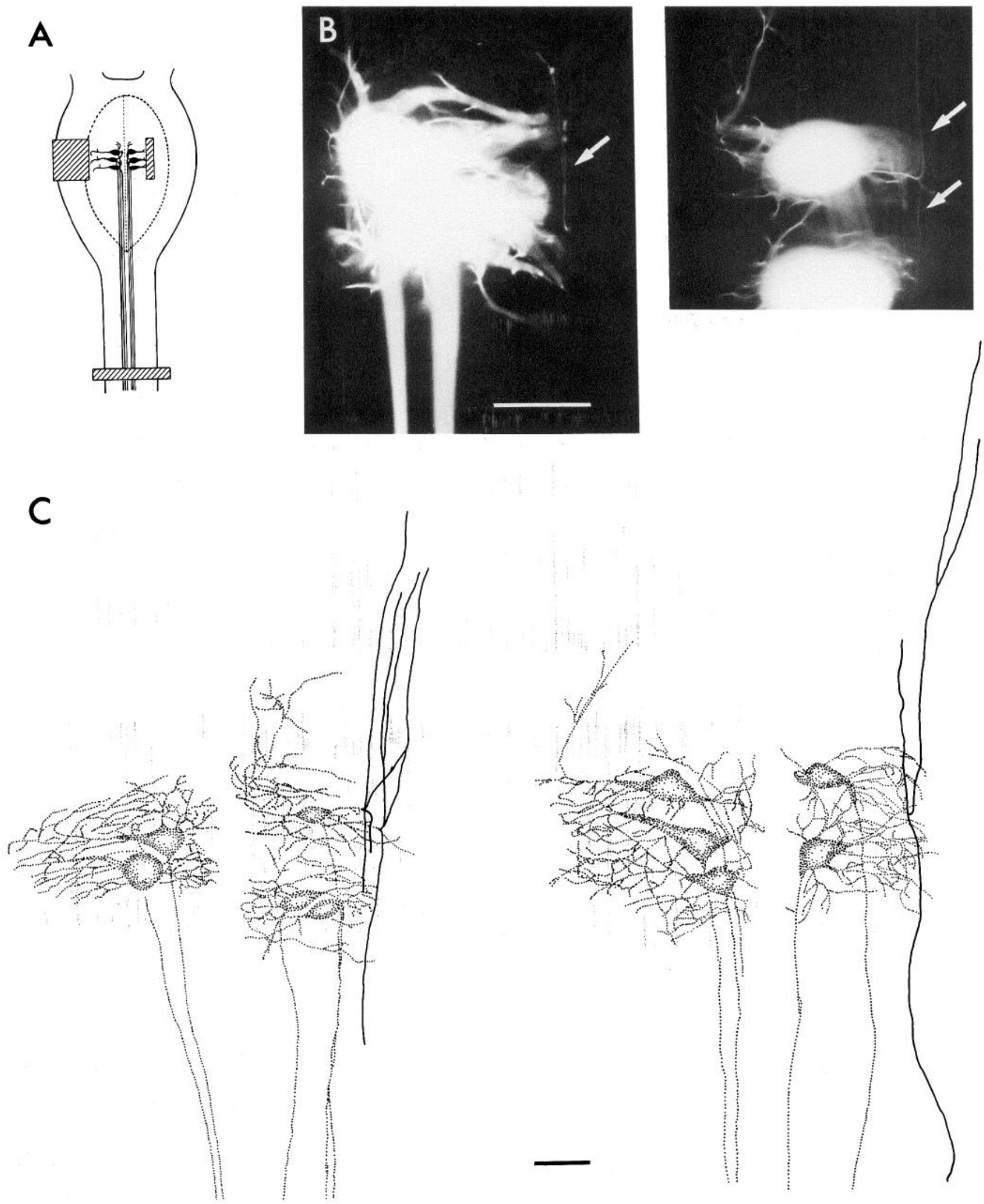

Figure 2. Response of $\mathrm{ABCs}$ to lateral dendrotomy following distant axotomy. A, Schematic diagram of the lamprey hindbrain showing sites of lateral dendrotomy (right side) and contralateral control ablation in the vicinity of the vestibular nucleus (hatched boxes). The site of distant axotomy in the spinal cord is also shown. B. Photographs of ABCs first subjected to distant axotomy and then dendrotomized $30 \mathrm{~d}$ later. Cells were filled $20 \mathrm{~d}$ after dendrotomy. Note multiple sprouts emerging from the dendritic lesion site (solid arrows). C, Tracings of ABCs from 2 animals subjected to distant axotomy $50 \mathrm{~d}$ prior to filling. In each case, ABCs on right received a lateral dendrotomy $30 \mathrm{~d}$ postaxotomy, while cells on left served as controls. Sprouts are traced in solid line. Note the linear, rostrocaudal trajectories of the sprouts and their site of origin at the dendritic lesion. Scale bars, $100 \mu \mathrm{m}$. 


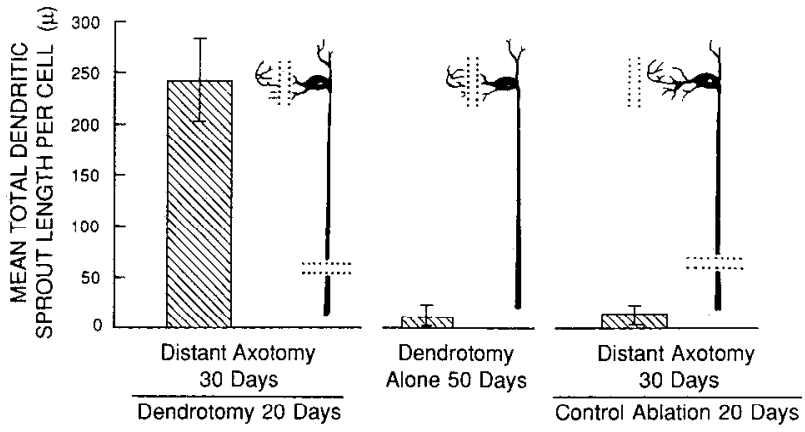

Figure 3. Total extent of dendritic sprouting: distant axotomy followed by dendrotomy versus control experiments. ABCs that had been subjected to distant axotomy $30 \mathrm{~d}$ earlier had significantly more dendritic sprouts ( $p<0.01$, Mann-Whitney $U$ test) by $20 \mathrm{~d}$ following dendrotomy ( $50 \mathrm{~d}$ after the initial distant axotomy) than lampreys subjected to control experiments. Schematic drawings next to each bar indicate lesions. $N$ for distant axotomy plus dendrotomy, 89 cells in 50 animals; for dendrotomy alone, 36 cells in 20 animals; distant axotomy plus control ablation, 46 cells in 22 animals.

difference between the abilities of axotomy and dendrotomy to evoke dynamic changes in dendritic form suggests that the integrity of the axon plays a special role in maintaining the morphological stability of the neuron.

\section{Dendritic sprouting is evoked by dendrotomy of axotomized $A B C s$}

We next tested the possibility that dendrotomy might influence the distribution of sprouting evoked by a previous axotomy. We examined the effect of dendrotomy on 89 ABCs in 50 animals in which the spinal cord had been transected at the level of the 5th gill $30 \mathrm{~d}$ previously ("distant axotomy"). In most animals, the dendrotomy was unilateral, with the dendrites of contralateral ABCs left intact to serve as controls. The area of the brain immediately lateral to the dendrites of the control cells was ablated (Fig. 2) to control for any indirect effects of the lesion, such as trauma to tissue immediately adjacent to the dendrites and the removal of vestibular inputs to ABCs. Since distant axotomy does not by itself evoke dendritic sprouting (Hall and Cohen, 1988), any dendritic sprouting seen following distant axotomy plus dendrotomy can be attributed to the effects of the dendrotomy lesion. The combination of distant axotomy followed by dendrotomy resulted in neuritic sprouting from the site of the dendritic lesion in many of the cells examined by 20 d postdendrotomy. Sprouting was rarely observed from contralateral control cells (Figs. 2C, 3).

The distribution of dendritic sprouting evoked by dendrotomy of $\mathrm{ABCs} 30 \mathrm{~d}$ after distant axotomy was distinctly different from that of cells subjected to "close" axotomy within $500 \mu \mathrm{m}$ of their somata (Hall and Cohen, 1988). Almost all of the sprouts evoked by dendrotomy originated from the site of the dendritic lesion (Fig. 4). By contrast, dendritic sprouts induced by close axotomy arose from sites distributed throughout the dendritic tree.

We next asked whether the site of the dendritic lesion determines the location of dendritic sprouting in neurons conditioned by a distant axotomy $30 \mathrm{~d}$ previously. In one group of conditioned animals we made a transverse lesion immediately rostral to the first pair of $\mathrm{ABCs}$. This operation removed rostrally projecting dendrites from both medial and lateral dendritic compartments and produced a significantly different distribution of

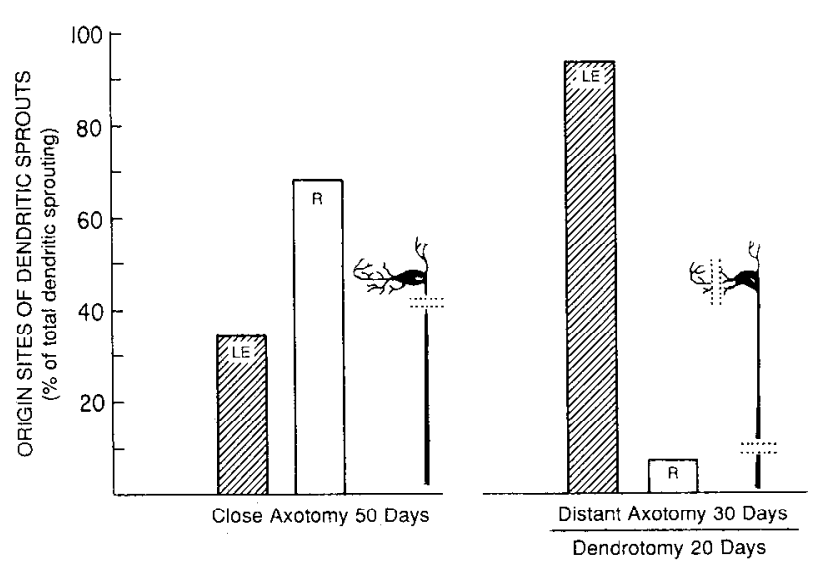

Figure 4. Histogram comparing the distribution of sprouting sites within the dendritic trees of $A B C s$ subjected to close axotomy versus $A B C s$ receiving distant axotomy followed by lateral dendrotomy. LE (lateral edge) refers to sprouts originating from the immediate vicinity of the dendrotomy lesion (within $10 \mu \mathrm{m}$ ) in cells subjected to distant axotomy plus dendrotomy; or the most lateral $50 \mu \mathrm{m}$ of the dendritic tree in cells subjected to close axotomy. $R$ refers to sprouts originating from the remaining dendrites (which are of roughly equal extent in the 2 groups). All cells were examined $50 \mathrm{~d}$ after axotomy. Note the highly significant $\left(p<0.01, \chi^{2}\right.$ test) bias towards sprouting from the dendritic lesion site following dendrotomy. $N$ : 149 sprouts for close axotomy, 49 sprouts for distant axotomy plus lateral dendrotomy.

dendritic sprouts from that evoked by lateral dendrotomy. Twenty-eight conditioned ABCs with 14 dendritic sprouts were examined $20 \mathrm{~d}$ after "rostral dendrotomy" (50 d after distant axotomy). Dendritic sprouts were evenly distributed between the medial ( 7 sprouts) and lateral ( 7 sprouts) dendritic compartments, and 11 of 14 sprouts originated from the vicinity of the lesion. No sprouting was evoked by this lesion in the 5 more posteriorly located ABCs examined that did not extend dendrites to the lesion site. These results suggest that sprouting occurs preferentially at or near the site of a dendritic lesion in axotomized $\mathrm{ABCs}$ irrespective of the site of dendrotomy.

\section{Factors controlling the amount and distribution of sprouting evoked by dendrotomy}

Further experiments were conducted in order to investigate the causes of sprouting evoked by dendrotomy in ABCs conditioned by distant axotomy $30 \mathrm{~d}$ previously. In particular, we wanted to know whether (1) the dendritic sprouting associated with dendrotomy results from the redistribution of an intrinsically limited amount of sprout material evoked by axotomy or (2) if dendrotomy evokes "extra" sprouting from the dendrotomy lesion site without affecting the amount of sprouting induced by axotomy elsewhere in the cell. To determine this, it was necessary to reaxotomize cells conditioned by distant axotomy at a site close enough to the soma for axonal sprouts to be visualized following somatic injection with Lucifer yellow. We therefore performed a set of experiments in which ABCs were first subjected to distant axotomy in the cord and then reaxotomized bilaterally at a site $750 \mu \mathrm{m}$ from the soma $30 \mathrm{~d}$ later (Fig. $5 A$ ). The site of reaxotomy was chosen so that significant amounts of both dendritic and axonal sprouting would be evoked by reaxotomy alone. A lateral dendrotomy was performed on one side at the time of the second axotomy. Ceils were filled with Lucifer yellow and examined $20 \mathrm{~d}$ after the reaxotomy and dendrotomy. This combination of lesions permitted us to assess in detail the effects of a dendritic lesion on the amount and 

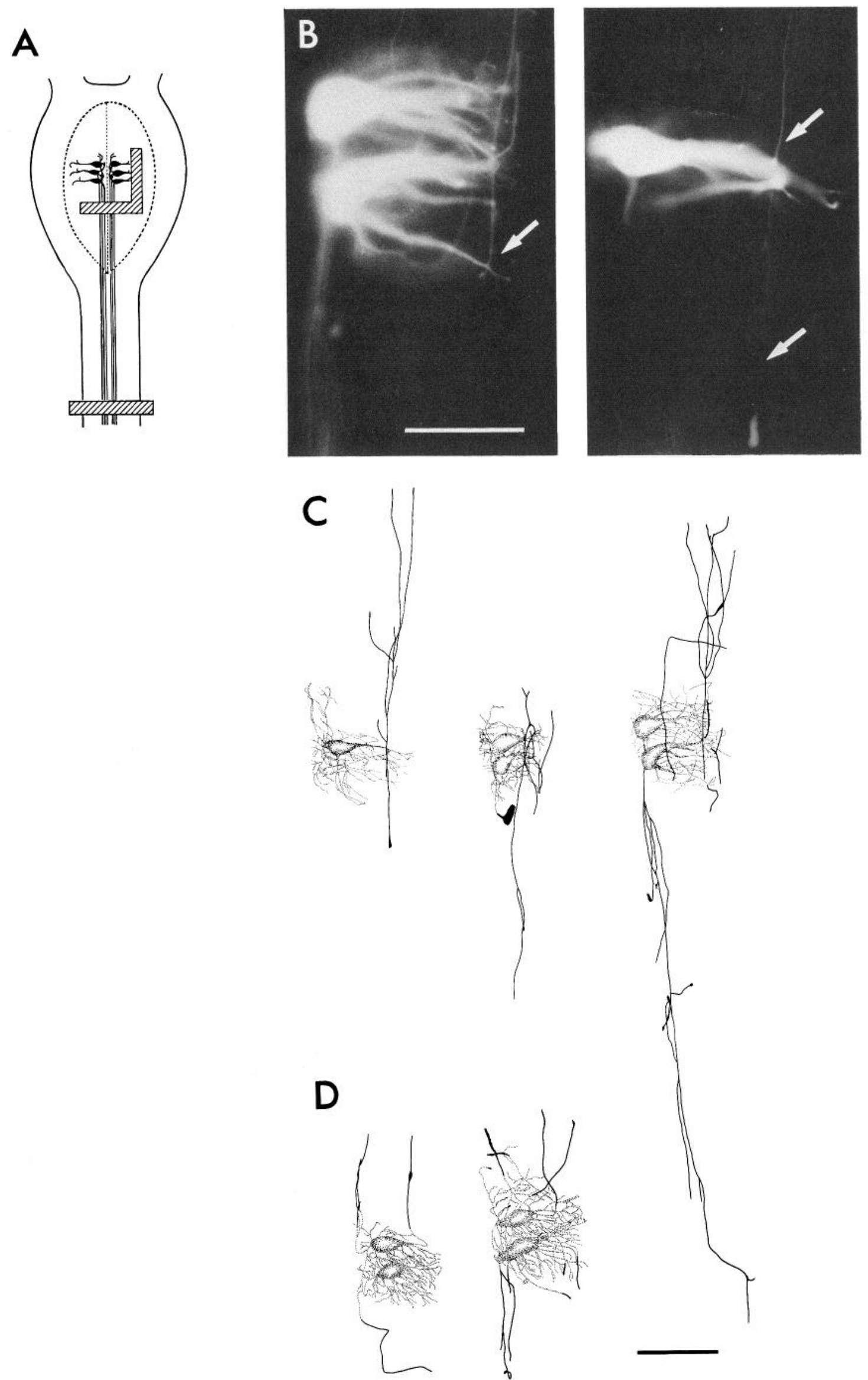
distribution of both dendritic and axonal sprouting evoked by axotomy.

Examples of $\mathrm{ABCs}$ treated according to this protocol are shown in Figure 5, and the results summarized in Figure 6. ABCs that had been subjected to both axotomy and dendrotomy showed somewhat larger total amounts of sprouting than did control cells subjected to axotomy alone, but this difference was not statistically significant. However, dendrotomy did cause a clear redistribution of the dendritic sprouts evoked by axotomy. Significantly more sprouting occurred at the site of dendrotomy than from the same region in control cells, while there was a significant reduction of sprouting from the rest of the dendritic tree. Thus, under the conditions of this experiment, dendritic sprouting occurred primarily from the site of the dendritic lesion at the expense of sprouting elsewhere in the dendrites. By contrast, the amount of axonal sprouting was not significantly affected by dendrotomy.

\section{Morphology of sprouts evoked by dendrotomy}

Sprouts evoked by dendrotomy of ABCs displayed morphological characteristics that distinguished them from the dendritic sprouting evoked by close axotomy as described in the accompanying paper (Hall and Cohen, 1988): (1) Sprouts evoked by dendrotomy invariably displayed highly linear trajectories despite the fact that they originated exclusively from the region immediately adjacent to the lesion site; these sprouts were always rostrocaudally oriented and often grew along the lesion for long distances (Fig. 2, $B, C$ ). This is quite different from the disruptive effect that the axonal lesion site has on the trajectories of sprouts evoked by close axotomy. (2) Sprouts evoked by dendrotomy were also somewhat finer in appearance than dendritic sprouts evoked by close axotomy alone. Perhaps because of this, the tips of sprouts evoked by dendrotomy often failed to fill with Lucifer yellow. Those tips that were visible were either tapered (no swelling) or spindle shaped and were thus similar to those seen on axially oriented dendritic sprouts evoked by close axotomy (Hall and Cohen, 1988). Virtually all of the dendritic sprouts evoked at the dendrotomy lesion site by a combination of axotomy and dendrotomy exhibited these characteristic trajectories and morphology (Fig. 5, $B, C$ ).

One possible reason for the distinct and highly stereotyped morphology of sprouts evoked by lateral dendrotomy is that the axially oriented lesion used to produce lateral dendrotomy may reinforce the axial guidance cues that sprouts follow in the hindbrain, whereas the transverse lesion used to produce close axotomy disrupts rostrocaudal sprout trajectories (Hall and Cohen, 1988). Much of the local sprouting evoked by dendrotomy in this situation appears to be due to the redistribution of the sprouting evoked by axotomy. Therefore, it is likely that the differences between sprouts associated with dendrotomy and axotomy are due to the differing nature of the environments produced by the dendritic and axonal lesions rather than to intrinsic differences in the nature of sprouts themselves.

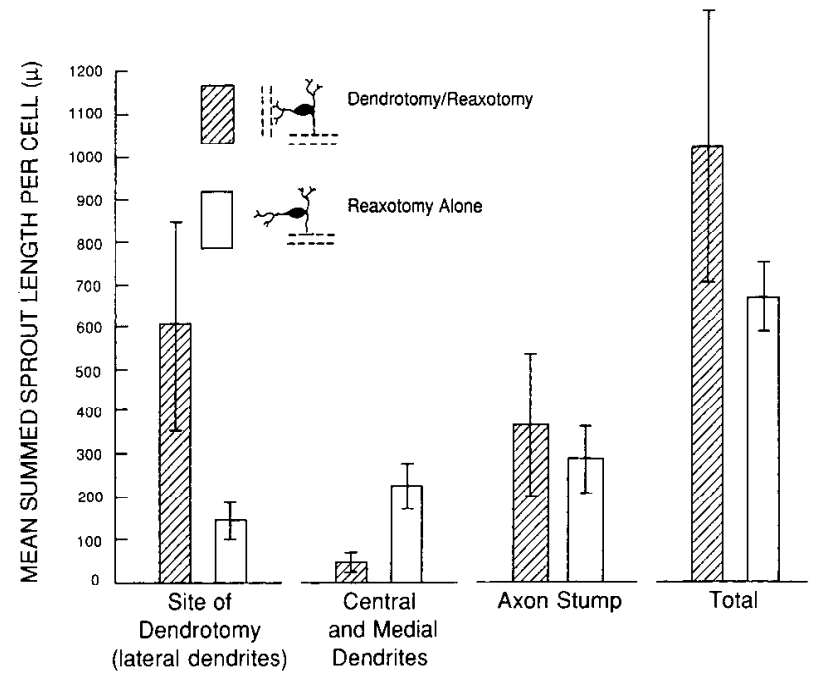

Figure 6. Histogram summarizing the amount and distribution of sprouting from ABCs subjected to distant axotomy and then reaxotomized $750 \mu \mathrm{m}$ from their somata. Cells subjected to reaxotomy plus dendrotomy (shaded bars) are compared with control cells (open bars) receiving reaxotomy alone. The amount of sprouting (given as the mean summed length of sprouting per cell, $\pm \mathrm{SE}$ ) emerging from within 10 $\mu \mathrm{m}$ of the dendrotomy site is compared with the amount of sprouting occurring from the most lateral $50 \mu \mathrm{m}$ of the dendrites in control cells. Other cellular compartments (central and medial dendrites, axon stump) are the same in control and experimental cells. The amount of sprouting from the site of dendrotomy is significantly greater than that seen from the equivalent lateral dendritic area in control cells $(p<0.001$, MannWhitney $U$ test), while the amount of sprouting from the rest of the dendritic tree (central and medial dendrites) is significantly less in dendrotomized cells than in control cells $(p<0.005$, Mann-Whitney $U$ test). The amount of axonal sprouting and the total amount of sprouting were not significantly different in the control and experimental groups $(p=0.18$ and 0.17 , respectively).

\section{Discussion}

\section{Competence to sprout and sprout distribution are controlled separately}

The results of this study can be briefly summarized as follows: (1) amputations of $\mathrm{ABC}$ processes must include axotomy in order for them to elicit neuritic sprouting anywhere in the cell, (2) dendrotomy of axotomized cells causes localized sprouting from the dendritic lesion, and (3) sprouting at the dendritic lesion is at least partly due to a redistribution of sprouting that would otherwise have occurred elsewhere in the cell. These findings can best be accounted for by proposing that separate cellular mechanisms are involved in controlling whether and where sprouting is evoked by injury. One of these mechanisms is responsible for "turning on" sprouting in ABCs after injury and depends upon axotomy. A separate group of factors controls where the sprouts emerge from the neuron by determining the relative attractiveness of different regions of the neuron as sites for sprout initiation.

Figure 5. Effect of dendrotomy on the distribution of sprouting evoked by axotomy. $A$, Schematic diagram illustrating the locations of lesion sites used in the experiment. ABCs that had been subjected to distant axotomy in the spinal cord were then reaxotomized $30 \mathrm{~d}$ later at a site in the hindbrain $750 \mu \mathrm{m}$ from their somata. Cells on the right side of the brain were also given a lateral dendrotomy at this time. All cells were then injected with Lucifer yellow $20 \mathrm{~d}$ later. $B$, Photographs of $\mathrm{ABCs}$ that received both reaxotomy and dendrotomy. Note sprouts emerging from the site of the dendritic lesion (arrows). Scale bar, $100 \mu \mathrm{m}$. C, Tracings of 3 sets of ABCs ( 2 of which are shown in $B$ ) subjected to reaxotomy and dendrotomy. Note that sprouts (solid lines) emerge predominantly from either the site of dendrotomy, axotomy, or both. $D$, Tracings of 2 sets of control cells subjected to reaxotomy alone. Note that sprouts emerge from all parts of the dendritic tree as well as the axon stump. 
The failure of dendrotomy to induce sprouting in otherwise intact neurons suggests that the ability of axotomy to turn on sprouting depends on properties of the axon that are not shared by the dendrites. Therefore, consequences of injury such as (1) depolarization at the injury site and (2) an influx of ions and other substances from the extracellular fluid at the lesion are unlikely to be the primary trigger for injury-induced sprouting in this system, as they probably occur with dendrotomy (Lucas et al., 1985) as well as axotomy (Borgens et al., 1980; Meiri et al., 1981). Rather, effects of injury that involve uniquely axonal properties, such as the loss of a substance that is normally retrogradely transported from a postsynaptic target or the loss of most of the neuronal cytoplasm with axotomy, are more plausible candidates for the signal by which injury evokes sprouting in this system.

It is clear from the results of this study that dendrotomy alone does not evoke axonlike sprouts from the dendrites similar to those produced by close axotomy. Sprouting was not observed even in the few ABCs that survived severe lesions close to their somata that removed nearly all of the lateral dendrites. It is possible that the extent of dendrotomy can never be sufficient to evoke any kind of sprouting; this might be expected if the signal for sprouting is simply the loss of a "threshold" amount of neuronal cytoplasm that can only occur with axotomy. However, it is still not certain that dendrotomy alone is incapable of inducing limited sprouting of dendrite-like processes in $\mathrm{ABCs}$, as limited dendritic regeneration within the confines of the original dendritic tree might be difficult to detect. Similarly, the results of our experiments cannot completely rule out a role for deafferentation in the control of dendritic sprouting from $\mathrm{ABCs}$. Our results indicate that the bilateral removal of afferents from the vestibular nuclei, which are one of the known major inputs to ABCs (Rovainen, 1979), does not elicit dendritic sprouting either alone or in conjunction with distant axotomy. Still, it is possible that lesions producing more extensive deafferentation of $\mathrm{ABCs}$ than those described here might cause dendritic sprouting in the absence of dendrotomy. Deafferentation does appear to induce dendritic sprouting in some systems, such as cockroach motor neurons (Pitman and Rand, 1982) and cricket auditory interneurons (Hoy et al., 1978). Our results do show that even slight injury to the dendrites in conjunction with axotomy readily produces sprouting from the immediate lesion site, whereas extensive lesions immediately adjacent to the dendrites evoke no dendritic sprouting if the dendrites are left intact. This strongly suggests that it is the dendrotomy itself, rather than the deafferentation caused by these lesions, that is directly responsible for dendritic sprouting in previously axotomized ABCs.

\section{Factors controlling sprout distribution}

\section{Role of the axon stump}

A number of instances have been described in which dendritic (or somatic) sprouting has been evoked by the axotomy of neurons close to their somata. This has been seen both in invertebrates (Murphy and Kater, 1980; Roederer and Cohen, 1983; Schacher and Proshansky, 1983) and vertebrates (Hall and Cohen, 1983; Yin and Selzer, 1984). In each of these cases, the axotomy site determined whether sprouting occurred from the axon stump alone or from the soma and/or dendrites, with axotomy close to the soma always producing sprouting from the soma or dendrites. It has been proposed that a neuron must retain a critical length of axon stump after axotomy in order to "funnel" new growth specifically into a regenerating axon
(Schacher and Proshansky, 1983; Roederer and Cohen, 1983), whereas loss of the axon stump results in the dispersal of sprouting to various sites in the soma or dendrites. However, our results clearly demonstrate that a short or missing axon stump is not a prerequisite for significant dendritic sprouting in lamprey $\mathrm{ABCs}$, as sprouting can be evoked by dendrotomy in cells that have very long ( $1 \mathrm{~cm}$ or more) axon stumps. Rather, dendritic sprouting appears to be the result of an increased attractiveness of the dendrites as sprouting sites.

The responses of lamprey ABCs to "intermediate" and "close" axotomy (Hall and Cohen, 1983, 1988) are also consistent with our conclusion that some factor other than damage to the axon stump is required for dendritic sprouting to occur. Intermediate axotomy always induces profuse sprouting from the axon stump as well as dendritic sprouts. The axon stump may even be an attractive site for sprouting following close axotomy if it does not degenerate completely. The $8: 1$ predominance of dendritic over axonal sprouting seen following close axotomy (Hall and Cohen, 1988) may be entirely due to a modest increase in the attractiveness of each dendritic tip as a sprouting site, especially in light of the large number of potential sprouting sites present in the dendrites versus the single site at the end of the proximal axon stump.

\section{Sprouting occurs at or near lesion sites}

The restriction of dendrotomy evoked sprouting to the immediate vicinity of the dendritic lesion suggests that injury increases the attractiveness of nearby areas of the cell as sprout initiation sites. The "sprout localizing effect" of dendrotomy is likely due to the local consequences of direct injury to the neuron. Two phenomena that might mediate this effect are local depolarization and influx of exogenous substances that must occur following injury. Although clear roles in the local promotion of sprouting have not been established for either of these, a number of studies suggest that an influx of inorganic ions, $\mathrm{Ca}^{2+}$ in particular, may be important in the response of neurons to injury. For instance, a large influx of $\mathrm{Na}^{+}$and $\mathrm{Ca}^{2+}$ has been demonstrated at the lesion site in axotomized lamprey Muller cells (Borgens et al., 1980). Modulation of this ionic influx with applied DC currents has been shown to alter the amount of local axonal degeneration in the proximal axon stumps (Roederer et al., 1983) and the extent of subsequent sprouting from the cut axon (Borgens et al., 1981). The presence of large $\mathrm{Ca}^{2+}$ currents in the growth cones of developing and regenerating neurites (Meiri et al., 1981; Grinvald and Farber, 1981) and the ability of $\mathrm{C} \mathrm{Ca}^{2+}$ influx to produce growth cone expansion and flattening in culture (Anglister et al., 1982) have also been reported. On the other hand, little is known about the role played by depolarization per se (if any) in neuronal plasticity following injury.

\section{Dendritic tips are inherently attractive sites for sprouting}

It is clear from the results of the accompanying paper (Hall and Cohen, 1988) that the proximity of a given locus to a lesion cannot be the only feature that determines its attractiveness as a sprouting sitc following axotomy of ABCs. For example, the dorsal aspect of the soma and the shaft of the axon stump do not sprout following intermediate axotomy, even though they are closer to the lesion site than the dendrites, which sprout routinely. Similarly, dendritic sprouting is only seen at dendritic tips following close and intermediate axotomy and not from the dendritic shafts. A similar preference for sprouting at dendritic tips has been observed in axolomized cricket giant interneurons 
(Roederer and Cohen, 1983). These observations suggest that dendritic tips are inherently more attractive sites for sprouting than any other location within the cell with the exception of the cut end of the axon stump.

It is interesting to compare the pattern of sprouting from axotomized $\mathrm{ABCs}$ with that of a group of unipolar neurons in the abdominal ganglia of Aplysia. Schacher and Proshansky (1983) have demonstrated that the presence of an axon stump following axotomy determines the distribution of subsequent sprouting from these neurons (LUQ cells) in culture. They made the following observations: (1) Cells cultured with axon stumps sprout only from the stump, even when it is very short ( 200 $\mu \mathrm{m}$ in length or less). (2) Cells cultured without axon stumps sprout from many sites on the soma, but the onset of sprouting is substantially delayed compared with cells cultured with axon stumps. (3) Large cells from mature ganglia do not sprout at all in culture unless an axon stump is present, in which case sprouting occurs solely from the cut end of the axon stump. These results clearly indicate that the cut end of the axon stump is the preferred site for sprouting in LUQ cells and that sprouting from the soma can only be done with some difficulty in young neurons and not at all in older cells.

A possible explanation of the differences between the pattern of sprouting evoked by axotomy in lamprey $\mathrm{ABCs}$ and that of the Aplysia LUQ neurons may lie in the fact that intact ABCs are multipolar, whereas the LUQ neurons are not. Thus, LUQ cells have only one preferred site for sprouting after axotomy, whereas $\mathrm{ABCs}$ have many. In both $\mathrm{ABCs}$ and LUQ neurons, neuritic sprouting always seems to occur preferentially from the ends of neuronal processes (i.e., dendritic tips and dendritic and axonal stumps). A possible reason for this is that the processes of mature neurons may retain the cytoskeletal specializations that permitted neurites to develop in the first place. Such specializations might well be nucleation centers for microtubules ("microtubule organizing centers") which appear to direct neurite formation in some systems (Spiegelman et al., 1979). These may then facilitate neuritic growth from process tips when the neuron is induced to sprout again following injury.

The limitation of sprouting to the tips and cut ends of dendrites and axons may explain an important difference between the effects of dendrotomy and axotomy in localizing sprouting near the lesion in ABCs. Sprouts evoked by dendrotomy always emerged within 20-30 $\mu \mathrm{m}$ from the lesion site, while dendritic sprouting could be evoked by an axotomy up to $1000 \mu \mathrm{m}$ away.

Figure 7. Schematic diagrams showing the importance of the locations of lesion sites and the predisposition of sprouts to originate at dendritic tips in determining the distribution of sprouting from $\mathrm{ABCs}$ following axotomy and dendrotomy. The sprout distribution resulting from each of the experiments described in this study and the companion paper (Hall and Cohen, 1988) are summarized. Stippled triangles at dendritic and axonal tips represent areas of the cell capable of supporting sprouting; hatched boxes represent lesion sites; black arrows indicate the relative amount and distribution of sprouting within the cell. Top row, Changing the location of the axotomy site changes the distribution of sprouting between dendrites and axon. At left, close axotomy causes the entire dendritic tree to be relatively close to the lesion site, providing a large number of attractive sites for sprout initiation. Most of the sprouting therefore occurs from the dendritic tree rather than from the axon stump, despite the continued attractiveness of the axon stump as a site for sprout initiation. As the site of axotomy is moved further from the dendritic tips, they become progressively less and less attractive individually relative to the axon stump until the total amount of sprouting from the dendrites becomes less than that occurring from the axon
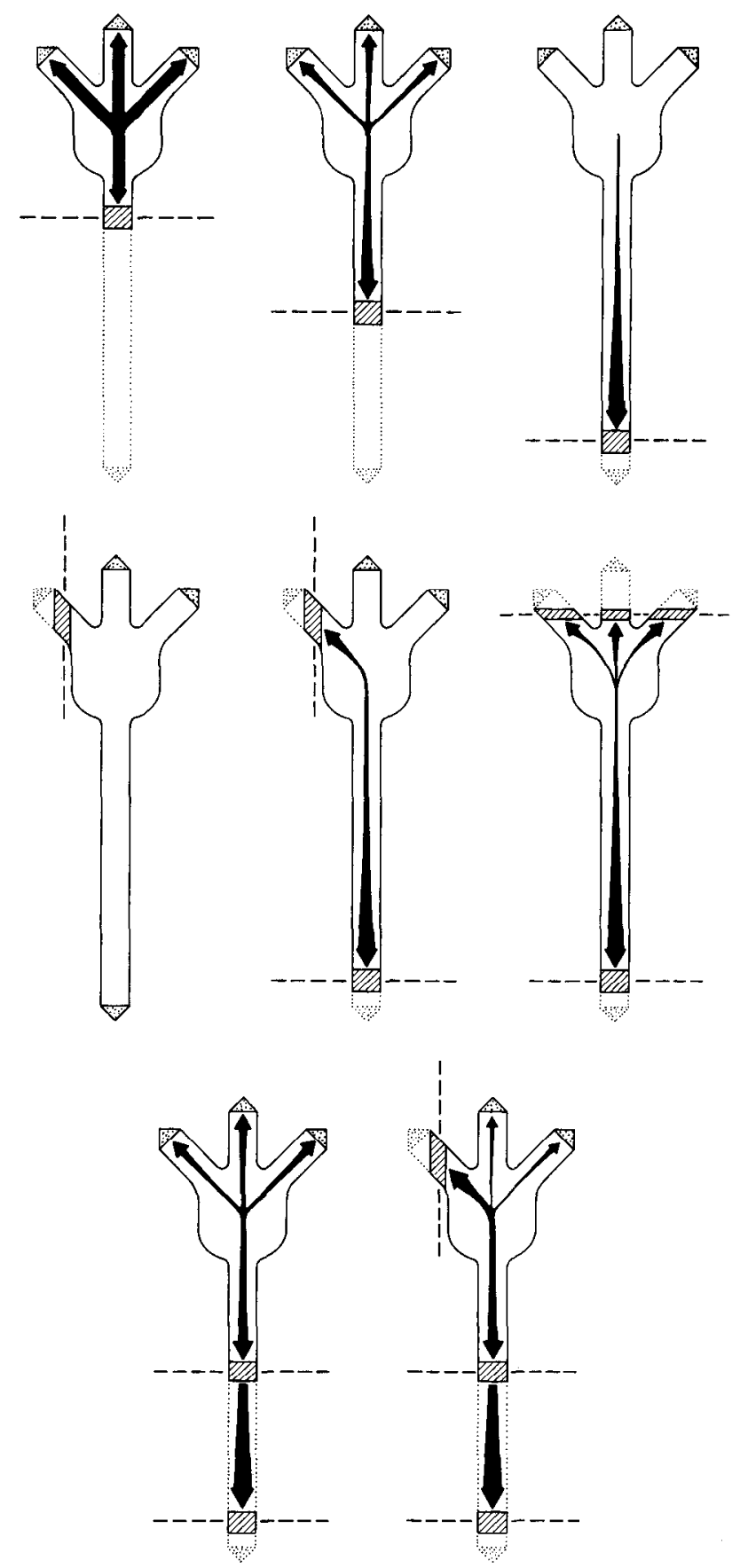

stump (top center). When the site of axotomy is moved far away from the dendritic tips (top right), sprouting occurs exclusively from the axon stump. Center row, Dendrotomy induces dendritic sprouting only in axotomized cells. Dendrotomy of intact ABCs (center left) does not induce dendritic sprouting, whereas dendrotomy of cells that have been subjected to distant axotomy (center and center right) results in sprouting from the site of dendrotomy. Bottom row, Dendrotomy causes a redistribution of sprouting within axotomized cells. When ABCs are reaxotomized at a site that results in both dendritic and axonal sprouting (bottom left), the presence of an additional dendrotomy (bottom right) causes more sprouting to occur from the site of dendrotomy at the expense of sprouting from other parts of the dendritic tree. The amount of axonal sprouting is not affected by the presence of dendrotomy. Thus, dendrites in the immediate vicinity of the lesion are clearly more attractive than other dendrites as sprouting sites even when the latter are quite close to the site of axotomy. This suggests that the increase in attractiveness caused by lesioning and proximity to the axotomy sitc is additive. 
This disparity may be due to the presence of many potential sprouting sites (i.e., cut dendrites and dendritic tips) located very close to the dendritic lesion. These might preemptively bind a sprout-initiating signal cmanating from the lesion sitc and thus prevent its wide distribution throughout the cell, especially if the signal is in short supply. Conversely, the lack of potential sprouting loci on the axon shaft and soma might permit the much greater spread of the sprout-initiating signal from the site of axotomy through the axon shaft and soma to the dendrites if axotomy occurs close enough to the soma.

\section{Competition between axonal and dendritic sites for sprouting}

The results presented in both this study and the accompanying paper (Hall and Cohen, 1988) suggest that axotomy induces $\mathrm{ABCs}$ to sprout at a limited rate which is not significantly affected by changing the site of axotomy or the presence of additional lesions in the cell. This means that the various sites within the cell must compete with one another to initiate sprouting, with the result that growth occurs preferentially from the most attractive site at the expense of other sites. We have shown here that the presence of a dendritic lesion combined with axotomy $750 \mu \mathrm{m}$ from the soma causes a diversion of sprouting to the site of dendrotomy and away from other parts of the dendritic tree. However, the presence of a dendritic lesion did not divert sprouting from the axon stump, suggesting that while the dendritic lesion is a more attractive sprouting site than other dendritic regions, it is not more attractive than the axonal lesion. Similarly, moving the site of axotomy from a point close to the soma to one further away renders intact dendrites relatively less attractive as sprouting sites and the axon stump more so (Hall and Cohen, 1988).

A hierarchy can be constructed on the basis of these observations (Fig. 7) in which lesioned dendrites or axons are the most attractive sites for sprouting, followed by intact dendritic tips situated close to the site of axotomy (i.e., following close axotomy), and then intact dendritic tips that are relatively distant from the site of axotomy (following intermediate or distant axotomy).

\section{Summary}

Our results suggest that 4 separate factors may be involved in determining whether sprouting occurs and where the sprouts emerge from injured $\mathrm{ABCs}$ in the lamprey:

1. An axotomy-dependent mechanism must be activated before sprouting can occur anywhere in the cell.

2. Axotomy causes the production of a preset, limited rate of sprouting that is not significantly changed by moving the lesion site or by the occurrence of additional lesions in the cell.

3. Only certain parts of the cell (intact dendritic tips or the cut ends of dendrites and axons) are able to sprout readily following injury.

4. A lesioned process is the most attractive site for sprouting, while areas of the cell close to the lesion are more attractive sites for sprouting than areas further away.

In both this study and the companion paper (Hall and Cohen, 1988) we have shown that the ability of $\mathrm{ABCs}$ to maintain separate axonal and dendritic identities is not absolute and that under certain circumstances the materials required for axonal regeneration appear to be diverted into the dendritic transport system. Our results suggest that the location of the lesion site is normally important in directing axonal regeneration correctly into the axon stump. The rules that govern the distribution of sprouting in injured $A B C$ s may well be relevant to the general questions of how a neuron first generates and then maintains both its overall form and the functional identity of dendrites and axons.

\section{References}

Aguilar, C. E., M. A. Bisby, E. Cooper, and J. Diamond (1973) Evidence that axoplasmic transport of tropic factors is involved in the regulation of peripheral nerve fields in salamanders. J. Physiol. (Lond.) 234: 449-464.

Anglister, L., I. C. Farber, A. Shahar, and A. Grinvald (1982) Localization of voltage sensitive calcium channels along developing neurites: Their possible role in regulating neurite elongation. Dev. Biol. 94: 351-365.

Borgens, R. B., L. F. Jaffe, and M. J. Cohen (1980) Large and persistent electrical currents enter the transected lamprey spinal cord. Proc. Natl. Acad. Sci. USA 77: 1209-1213.

Borgens, R. B., E. Roederer, and M. J. Cohen (1981) Enhanced spinal cord regeneration in lamprey by applied electric fields. Science 213 : 611-617.

Grinvald, A., and I. C. Farber (1981) Optical recording of $\mathrm{Ca}^{++}$action potentials from growth cones of cultured neurons using a laser microbeam. Science 212: 1164-1169.

Hall, G. F., and M. J. Cohen (1983) Extensive dendritic sprouting induced by close axotomy of central neurons in the lamprey. Science 222: 518-521.

Hall, G. F., and M. J. Cohen (1988) The pattern of dendritic sprouting and retraction induced by axotomy of lamprey central neurons. $J$. Neurosci. 8: 3584-3597.

Hall, G. F., A. Poulos, and M. J. Cohen (1987) Ultrastructure of dendritic sprouts seen following close axotomy of giant central neurons in the lamprey. Soc. Neurosci. Abstr. 13: 280.12.

Hoy, R. R., G. B. Casaday, and S. Rollins (1978) Absence of auditory afferents alters the growth pattern of an identified auditory interneuron. Soc. Neurosci. Abstr. 4: 115.

Lucas, J. H., G. W. Gross, D. G. Emery, and C. R. Gardner (1985) Neuronal survival or death after dendrite transection close to the perikaryon: Correlation with electrophysiologic, morphologic and ultrastructural changes. Central Nervous System Trauma (American Paralysis Assoc.) 2(4): 231-257.

Mackler, S. A., H.-S. Yin, and M. E. Selzer (1986) Determinants of directional specificity in the regeneration of lamprey spinal axons. J. Neurosci. 6: 1814-1821.

Meiri, H., M. E. Spira, and I. Parnas (1981) Membrane conductance and action potential of a regenerating axonal tip. Science $211: 709$ 712.

Murphy, A. D., and S. B. Katcr (1980) Sprouting and functional rcgeneration of an identified neuron in Helisoma. Brain Res. 186: 251 272.

Pitman, R. M., and K. A. Rand (1982) Neural lesions can cause dendritic sprouting of an undamaged adult insect motoneurone. J. Exp. Biol. 96: 125-130.

Roederer, E., and M. J. Cohen (1983) Regeneration of an identified central neuron in the cricket. I. Control of sprouting from soma, dendrites, and axon. J. Neurosci. 3: 1835-1847.

Roederer, E., N. H. Goldberg, and M. J. Cohen (1983) Modification of retrograde degeneration in transected spinal axons of the lamprey by applied DC current. J. Neurosci. 3: 153-160.

Rovainen, R. M. (1979) Electrophysiology of vestibulospinal and vestibuloreticulospinal systems in lampreys. J. Neurophysiol. 42: 745765.

Schacher, S., and E. Proshansky (1983) Neurite regeneration by Aplysia neurons in dissociated cell culture: Modulation by Aplysia hemolymph and the presence of the initial axonal segment. J. Neurosci. 3: 2403-2413.

Spiegelman, B. M., M. A. Lopata, and M. W. Kirschner (1979) Aggregation of microtubule initiation sites preceding neurite outgrowth in mouse neuroblastoma cells. Cell 26: 253-263.

Yin, H.-S., S. A. Mackler, and M. E. Selzer (1984) Directional specificity in the regeneration of lamprey spinal axons. Science $224: 894$ 896. 